\title{
PERSPECTIVES
}

\section{Up (to) date for Australian Toxicology and Toxinology guidelines}

\section{Authors:}

1. Dr Angela L Chiew BSci (Med) MBBS, PhD, FACEM; Clinical Toxicologist, Prince of Wales Hospital, Clinical Toxicologist, NSW Poisons Centre

2. Professor Nicholas A Buckley MD, FRACP; Chair of Toxicology and Toxinology Expert group, Therapeutic Guidelines Ltd., Professor of Clinical Pharmacology, University of Sydney, Clinical Toxicologist, NSW Poisons Centre

3. Professor Andis Graudins MBBS, PhD, FACEM, FACMT; Director, Monash Toxicology Unit, Monash Health, Clinical Toxicologist, Victorian Poisons Information Centre 4. Dr Venita Munir MBBS, FACEM, Assoc Deg PWE; Editor, Therapeutic Guidelines Ltd.

Corresponding author: Dr Angela L Chiew

Department of Emergency Medicine

Prince of Wales Hospital

Barker Street Randwick 2031

Email: angela.chiew@health.nsw.gov.au

Authors contribution statement: AC, NB, AG and VM drafted and edited the article and all authors approved the final version.

This is the author manuscript accepted for publication and has undergone full peer review but has not been through the copyediting, typesetting, pagination and proofreading process, which may lead to differences between this version and the Version of Record. Please cite this article as doi: 10.1111/1742-6723.13663

This article is protected by copyright. All rights reserved. 


\title{
Conflict of interest:
}

AC and AG: are section editors for the EMA

AC, NB and AG: were a part of the expert panel for eTG complete, Toxicology and Toxinology guideline.

VM: is an editor for Therapeutic Guidelines

Keywords: poisoning, toxicology, guidelines, management

Short running title: Toxicology and Toxinology Guidelines

\begin{abstract}
:
Poisoned patients commonly present to emergency departments. The optimal management of these patients is constantly evolving as new evidence emerges. Textbooks cannot be updated regularly enough to incorporate these changes, and their advice may not reflect the current practice of experts. Clinicians treating poisoned patients need an up-to-date, accessible resource to aid their management decisions. The updated eTG complete, Toxicology and Toxinology guideline, represents a consensus of contemporary evidence and expert views from across Australia. Important key changes include: updated advice on decontamination, particularly use of activated charcoal; stepwise escalation in supportive care, including guidance on the most appropriate inotropes for each agent; and antidote recommendations for specific poisoning scenarios. Toxicology and Toxinology covers more than 100 poisoning topics and offers more detailed risk assessment, management, and
\end{abstract}


disposition advice. It is a valuable evidence-based resource for the management of poisoned patients.

Keywords: poisoning, toxicology, guidelines, management

\section{eTG complete: Toxicology and Toxinology guidelines - rebuilding and rebranding}

While all therapeutic guidelines need regular updates as evidence changes, the issues for Australian toxicology guidelines were far more complex and thus required a complete overhaul. Therapeutic Guidelines' Toxicology and Wilderness was last revised in 2012 and lacked coverage of many important poisonings. Its format as a small handbook meant the content and style were difficult to update. In common with all textbooks, it progressively became out of date, and in some respects, did not reflect the needs of our patients or current expert advice.

The new Toxicology and Toxinology guideline now represents the consensus of contemporary expert views from across Australia. The expanded expert group comprised nine clinical toxicologists from five states (seven emergency physicians, two physicians), four specialist pharmacists from the four Australian Poisons Information Centres, and one general practitioner working in both metropolitan and remote locations. The editorial team at Therapeutic Guidelines brought their decades of experience to ensure the guideline has clear structure and wording to reduce ambiguity and misinterpretation.

Toxicology and Toxinology is accessible through subscription online (eTG complete website), and via the downloadable eTG complete application, which can be used both online and offline. All Australian state health departments (and limited New Zealand regions) have access via government-sponsored links.

This article is protected by copyright. All rights reserved. 
The guideline has roughly doubled in size, giving specific, evidence-based advice for many drugs and toxic agents. Each topic is backed by up-to-date reference lists [1]. Therapeutic Guidelines is committed to frequent updates of its online content as required (across the whole of eTG complete). The digital format of Toxicology and Toxinology means that topics can be updated on a quarterly basis if required, or more rapidly if there is an urgent practicechanging amendment.

\section{Key updates: supportive care, gastrointestinal decontamination and antidotes}

The updated Toxicology and Toxinology guideline emphasises the roles of appropriate stepwise escalation in supportive care, and antidote use for specific poisoning scenarios. An example is the management of cardiovascular toxicity due to poisoning. The guideline recommends a stepwise approach to inotropic support, with catecholamines first-line, and high-dose insulin euglycaemia therapy (HIET) as second-line inotropic support.

Extracorporeal membrane oxygenation (ECMO) can also be considered for patients with drug-induced hypotension refractory to maximal inotropic support.

The guideline recognises that responses to antidotal therapy may not be identical across drug classes. For example, cardiovascular toxicity due to calcium channel blocker (CCB) poisoning varies across the drug class. Verapamil and diltiazem primarily cause cardiogenic shock and are more likely to benefit from HIET. Dihydropyridine CCBs generally cause vasodilatory shock and are consequently more likely to require vasopressor support, rather than inotropes.

Recommendations for serum alkalinisation includes more detailed advice for the treatment of drug-associated QRS widening and sodium-channel blockade. Serum alkalinisation using hypertonic sodium bicarbonate (8.4\%) and mechanical hyperventilation are recommended; however, repeated intravenous bolus dosing of sodium bicarbonate is advised, rather than 
continuous intravenous infusion. It is noted that some drugs that cause QRS widening and sodium-channel blockade (eg propranolol, hydroxychloroquine, lamotrigine) are less likely to respond to serum alkalinisation. The guideline suggests titration of sodium bicarbonate to a target serum $\mathrm{pH}$, and maximum doses for sodium bicarbonate to avoid hypernatraemia and excessive alkalaemia. It also emphasises the importance of concurrent mechanical hyperventilation to assist in maintaining alkalaemia, as well as cautioning about the risk of hypokalaemia associated with alkalaemia.

The perceived role of activated charcoal (AC) for gastrointestinal decontamination in emergency care has diminished over the past 10 to 15 years. This may be, in part, due to a misconception that there is only a 1-hour window for administration of $\mathrm{AC}$ after a toxic ingestion. The updated guideline highlights that $A C$ may be of benefit for up to two hours after ingestion of an immediate-release formulation, and at least four hours after ingestion of a modified-release formulation, and even later in patients with severe poisoning or very large drug ingestions. This specific advice acknowledges that more modified-release formulation drugs are being developed, and there is a recent increasing trend for very large or massive drug ingestions. Massive monodrug ingestion or large polydrug ingestion can cause slowed gastrointestinal motility, delayed drug absorption and prolonged duration of toxicity. Toxicology and Toxinology also highlights the benefits of specific antidotes in drug poisonings and toxidromes (toxic syndromes). For example, physostigmine (if available) is recommended over benzodiazepines for agitated delirium in anticholinergic toxidrome. Notably, it is a receptor-specific antidote that can reverse the central nervous system effects of anticholinergic toxidrome. Its advantages also include a lower risk of over-sedation and reduced need for intubation of agitated, delirious patients. 
Octreotide is another example of an underused specific antidote and is recommended for all patients with sulfonylurea drug poisoning and hypoglycaemia. It reduces endogenous insulin secretion in response to intravenous glucose replacement.

Another setting in which the guideline is ahead of current textbooks is the dosing of digoxin immune antibody fragments (digoxin immune Fab) for digoxin toxicity. Practice-changing evidence has emerged for the management of digoxin toxicity, which recognises the management for acute digoxin poisoning is different to that for chronic digoxin accumulation [2]. Previously, recommended doses of digoxin immune Fab were based on a patient's serum digoxin concentration. This often resulted in excessive dosing of digoxin immune Fab. In chronic digoxin accumulation most patients will not require treatment with digoxin immune Fab.

Based on recent studies, most patients with chronic digoxin accumulation only require one vial of digoxin immune Fab to rapidly bind all free serum digoxin. Furthermore, management for acute digoxin poisoning in patients not in cardiac arrest has significantly changed; evidence supports titrating doses of two vials of digoxin immune Fab, and repeating doses if toxicity recurs [2].

For many years, paracetamol has been the commonest drug poisoning in Australia. Management had changed very little, although there have always been problems with the traditional three-bag acetylcysteine infusion regimen. These included a high incidence of adverse effects, and inadequate dosage for high risk poisonings (eg massive ingestions, ingestion of modified-release formulations). Emerging evidence has led to significant changes in the management of paracetamol poisoning, including a two-bag, 20-hour acetylcysteine infusion regimen, which causes fewer severe reactions [3]. Higher doses of acetylcysteine are recommended for those at high risk of acute liver injury (eg high initial serum paracetamol concentrations, large ingestions of modified-release formulations). 
Different management is also recommended for ingestions of immediate-release versus modified-release formulations [4,5]. Toxicology and Toxinology guidelines are aligned with the 2019 national guidelines for the management of paracetamol poisoning [6].

Another good example of the limitations of textbooks, in keeping up to date with current guidance, is the management of Australian snake bite. Snake bite diagnosis and management is constantly evolving in response to evidence emerging from the Australian Snakebite Project [7]. Just a decade ago, toxicology and toxinology textbooks recommended: the use of venom detection kits for diagnosis; administration of five ampoules of snake antivenom as standard practice; administration of further doses of snake antivenom in response to a trend in blood investigations; and avoidance of coagulation factor replacement, even in bleeding patients $[8,9]$. These recommendations have now been refuted by evidence. The updated snake bite management guideline focuses on early recognition of the clinical signs of systemic envenoming, followed by administration of snake antivenom(s) that cover(s) the possible snake species or group thought to be responsible. This is based on which snake species or group(s) are endemic to the location, and the patient's envenoming syndrome.

\section{Benefits for education and training in emergency medicine}

Compared to the previous versions, Toxicology and Toxinology offers clearer guidance, with each topic including advice on risk assessment, treatment, and observation and disposition. Importantly, high-risk ingestions now have detailed recommendations for when to seek expert advice. For drug treatments, more detailed information is provided for dosage, administration, and suggested endpoints. This makes the guideline a useful resource for cases where a protocol is not readily available for uncommon treatments and antidotes. 
Toxicology and Toxinology offers considerably different management advice compared to what is available in toxicology textbooks. This highlights how important the guideline can be to non-expert clinicians (eg junior doctors, rural and remote doctors) who use these contemporary consensus expert guidelines as a primary resource. The updated guideline also offers emergency physicians a superior toxicology resource over textbooks when providing advice on the acute management of the poisoned patient. The electronic format of eTG complete, with its ability to update Toxicology and Toxinology more readily, more frequently and more urgently, if required, is a great advantage of this resource. Ideally, it should be integrated into the Australasian College for Emergency Medicine's training syllabus, as it offers a more current, updatable, and evidence-based approach.

\section{References}

1. Toxicology and Toxinology [published 2020 Aug]. In: eTG complete [digital]. Melbourne: Therapeutic Guidelines Limited; 2020 Sept. <https://www.tg.org.au>

2. Chan BS, Isbister GK, O'Leary M, Chiew A, Buckley NA. Efficacy and effectiveness of anti-digoxin antibodies in chronic digoxin poisonings from the DORA study (ATOM1). Clin Toxicol (Phila). 2016;54(6):488-494.

3. Wong A, Graudins A. Simplification of the standard three-bag intravenous acetylcysteine regimen for paracetamol poisoning results in a lower incidence of adverse drug reactions. Clin Toxicol (Phila) 2016;54(2):115-9.

4. Chiew AL, Isbister GK, Kirby KA, Page CB, Chan BSH, Buckley NA. Massive paracetamol overdose: an observational study of the effect of activated charcoal and increased acetylcysteine dose (ATOM-2). Clin Toxicol (Phila) 2017;55(10):1055-65. 
5. Chiew AL, Isbister GK, Page CB, Kirby KA, Chan BSH, Buckley NA. Modified release paracetamol overdose: a prospective observational study (ATOM-3). Clin Toxicol (Phila). 2018:1-10.

6. Chiew AL, Reith D, Pomerleau A, Wong A, Isoardi KZ, Soderstrom J, et al. Updated guidelines for the management of paracetamol poisoning in Australia and New Zealand. Med J Aust 2019.

7. Johnston Cl, Ryan NM, Page CB, Buckley NA, Brown SG, O'Leary MA, et al. The Australian Snakebite Project, 2005-2015 (ASP-20). Med J Aust 2017;207(3):119-25.

8. Murray L, Daly F, Little M, Cadogan M. Toxicology handbook. Elsevier Australia; 2011.

9. White J. CSL Antivenom Handbook. 2nd ed. Melbourne: CSL Ltd; 2001. 


\title{
PERSPECTIVES
}

\section{Up (to) date for Australian Toxicology and Toxinology guidelines}

\section{Authors:}

1. Dr Angela L Chiew BSci (Med) MBBS, PhD, FACEM; Clinical Toxicologist, Prince of Wales Hospital, Clinical Toxicologist, NSW Poisons Centre

2. Professor Nicholas A Buckley MD, FRACP; Chair of Toxicology and Toxinology Expert group, Therapeutic Guidelines Ltd., Professor of Clinical Pharmacology, University of Sydney, Clinical Toxicologist, NSW Poisons Centre

3. Professor Andis Graudins MBBS, PhD, FACEM, FACMT; Director, Monash Toxicology Unit, Monash Health, Clinical Toxicologist, Victorian Poisons Information Centre

4. Dr Venita Munir MBBS, FACEM, Assoc Deg PWE; Editor, Therapeutic Guidelines Ltd.

\author{
Corresponding author: Dr Angela L Chiew \\ Department of Emergency Medicine \\ Prince of Wales Hospital \\ Barker Street Randwick 2031
}

Email: angela.chiew@health.nsw.gov.au

Authors contribution statement: AC, NB, AG and VM drafted and edited the article and all authors approved the final version.

This article is protected by copyright. All rights reserved. 


\section{Conflict of interest:}

$A C$ and $A G$ : are section editors for the EMA

AC, NB and AG: were a part of the expert panel for eTG complete, Toxicology and Toxinology guideline.

VM: is an editor for Therapeutic Guidelines

Keywords: poisoning, toxicology, guidelines, management

Short running title: Toxicology and Toxinology Guidelines

This article is protected by copyright. All rights reserved. 


\section{University Library}

\section{- M M N E R VA A gateway to Melbourne's research publications}

Minerva Access is the Institutional Repository of The University of Melbourne

\section{Author/s:}

Chiew, AL;Buckley, NA;Graudins, A;Munir, VL

Title:

Review article: Up (to) date for Australian Toxicology and Toxinology guidelines.

Date:

2021-02

Citation:

Chiew, A. L., Buckley, N. A., Graudins, A. \& Munir, V. L. (2021). Review article: Up (to) date for Australian Toxicology and Toxinology guidelines.. Emerg Med Australas, 33 (1), pp.6-8. https://doi.org/10.1111/1742-6723.13663.

Persistent Link:

http://hdl.handle.net/11343/276545 